\title{
SIMULATION OF PRODUCTION PROCESSES IN COMBINATION WITH TRACEABILITY
}

\author{
Horea Mizgan ${ }^{1}$, Ovidiu Ambruş², Macedon Ganea ${ }^{3}$ \\ ${ }^{I}$ INTELIFORM Co \\ 21 Moşniţei Street, Timişoara, Romania \\ horea.mizgan@gmail.com, \\ ${ }^{2}$ TRW Co, Timişoara \\ 16C Măcin Street, Timişoara, Romania \\ ovidiuambrus@yahoo.ro \\ ${ }^{3}$ University of Oreadea, \\ 1 Universitătii Street, Oradea, Romania \\ macedonganea@yahoo.com
}

\begin{abstract}
The TTM (Total Traceability Management) is becoming a mandatory process requested by all OEMs (Original Equipment Manufacturing) as a system to be deployed by the relevant suppliers. Process parameters from the machines are gathered by the TTM software and based on a defined algorithm the product is receiving new statuses, as physical and software characteristics. Given the high complexity of the current process flows, the highly automated lines and cells, the increased amount of process parameters, the necessity of synchronizing various production steps, it is needed that a simulation process to be done in advance of the full process deployment. The paper work reviews both the mathematical apparatus that underlies the simulation, and one of the various available simulation programs in academia. It is used one of the most usual software for simulation SIMQuick which is based on statistical methodologies. SIMQuick performs a virtual simulation of the process being used in various areas of activity including production in automotive, automation, supply chain processes and services, providing the valuable data for the behavior of the process and providing the premises for layout, flows, and resources improvements. The supply chain software ERP requirements with TTM technical inputs and outputs are the data pillars for the simulation which are presented as a result of the research.
\end{abstract}

Keywords: Simulation, Total Traceability Management, Artificial Intelligence, Predictive Maintenance

\section{Introduction}

Simulation is a method of verifying hypotheses, given that the direct implementation of these hypotheses could be irreversible or could negatively affect processes. The paper work reviews both the mathematical apparatus that underlies the simulation, and one of the various available simulation programs in academia. Now the challenge is to combine the simulation with the traceability in the purpose of quick response at simulation data and automation of entire verifying process. The simulation of production processes is based, as a general principle on probability theory.

Probability theory studies random phenomena, those events that if we reproduce repeatedly take place differently each time, and the result cannot be anticipated.

(C) 2021 Published by University Press. This is an open access article under the CC BY-NC-ND license (http://creativecommons.org/licenses/CC-BY-4.0/) 


\section{The mathematical apparatus associated with simulation}

The frequency of events can be described by the following formula:

$$
h(E)=\frac{M}{n}
$$

where $M$ represents the number of occurrences $E$ in the case of $n$ trials.

Mean value and dispersion: the random variable $X$ that takes the values $\boldsymbol{x}_{\boldsymbol{i}}$ and the corresponding probabilities $\mathbf{p}_{\mathbf{i}}$ are expressed by the occurrences:

$$
\mathbf{M}(\mathbf{X})=\sum_{\mathbf{i}=\mathbf{1}}^{\infty} \mathbf{x}_{\mathbf{i}} \mathbf{p}_{\mathbf{i}}
$$

Bernoulli statement (law of large numbers): the probability that the modulus of the difference between the relative frequency of event $\mathrm{E}$ in the case of $\mathrm{n}$ experiments ( $\mathrm{n}$ large enough) and the probability $\mathrm{p}$ of event $\mathrm{E}$ is less than $\varepsilon$ positive, arbitrarily small, is approximately equal to one:

$$
P\left(\left|\frac{m}{n}-p\right|<\epsilon\right) \geq 1-\frac{1}{4 \epsilon^{2} n}
$$

The most common mathematical modeling of statistical distributions of sets is Gaussian distribution (normal) [1]:

$$
p(x)=\frac{1}{a \sqrt{2 \pi}} e^{-\frac{(x-b)^{2}}{2 a^{2}}}
$$

where $\mathrm{p}(\mathrm{x})$ is the distribution density, the mean $\mu=\mathrm{b}$ and $\sigma^{2}=\mathrm{a}^{2}, \sigma^{2}$ being the dispersion.

In this case, the distribution function (Gaussian integral) is as follows:

$F(x)=\int_{-\infty}^{x} p(t) d t=\frac{1}{a \sqrt{2 \pi}} \int_{-\infty}^{x} e^{-\frac{(t-b)^{2}}{2 a^{2}}} d t$

\section{The simulation steps}

Steps to follow to simulate a process, as described in the literature [2] are:

a) Identifying the process to be studied and defining the improvement objectives;

b) Identifying the process aspects that vary;

c) Construction of a process flow scheme;

d) Collecting the necessary data for modeling the process;

e) Using / choosing statistical distribution elements suitable for the built model;

f) Building the model and introducing it in the chosen simulation program;

g) Determining the duration of the simulation and the number of simulations, so as to fit as well as possible with the reality of the process;

h) Running the simulation;

i) Validation of the simulation - comparison with the real process and correction of possible "deviations";

j) Analyze the results once the simulation has been accepted.

\section{The simulation program}

SimQuick [3] is a simulation program based on the Excel platform, used to model and simulates a wide variety of processes, such as, [4-7]:

- Inventory and distribution systems (e.g. stores, warehouses and combinations thereof) $[8,9]$;

- Production processes (e.g. assembly lines, processes involving parts series) $[10,11]$;

- Projects for which the time to meet certain conditions is not determined [12].

The program is used successfully in the university environment, as an alternative to the extremely complex and expensive simulation programs existing on the market.

\section{Creating the model}

SimQuick provides the programmer with five different blocks, called "elements", which can be arranged in a huge number of combinations, these combinations being the models to be simulated. The five elements are grouped under the headings:
1. Inputs;
2. Exits;
3. Stocks / buffers;
4. Workstations;
5. Decision points.

The first step in using SimQuick is to create / draw a process flow or logical scheme of the process in real life, using the aforementioned blocks:

Inputs - the place where objects enter the process. There may be examples of docks at a warehouse, a door at the store, etc $[13,14]$.

Stocks / buffers - the place where objects are stored, between operations. These can be locations in a warehouse, a place where people wait in line, etc., and it is necessary to specify the maximum storage capacity of each storage place.

Workstations - places where added value is produced in the process. These can be representations of processing machines in factories, cash registers / cash registers in shops, computers in a network, etc., and here the working time on each object at the respective workstation must be specified using the statistical distribution [15].

Decision points - the places where it is decided whether an object goes in one direction or another, based on imposed conditions. They can be specified with this simulator up to 10 directions for each object. The decision points can be the result of a quality control, the result of the verification of a processing, etc., in which case the rule according to which this selection must be made has to be specified, using the statistical distribution.

Outputs - the places where objects exit the process. These can be representations of the output docks from a warehouse, and here it must be specified how many objects leave the process at a given time and at what time intervals, using statistical distribution methods [16-19]. 
Types of statistical distributions (histograms) used by the simulation program [3] are:

a. Bell-shaped distribution - most results centered in the middle of the range. In this situation, the model will generate random numbers in the specified range (Fig. 1);

b. Right centered distribution - In this situation, the model will generate random numbers in the specified range, having this statistical distribution;

c. Equal distribution - the real values of the process are substantially equal. In this situation, the model will generate random numbers in the specified range, with the minimum and maximum specified;

d. Specific distribution - different minimum / maximum values can be set for up to 10 different possible results.

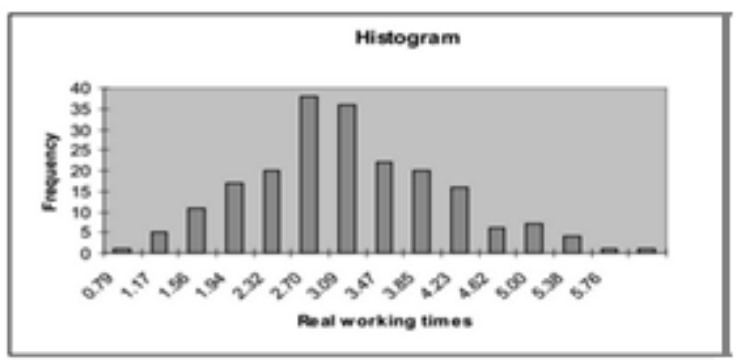

a)

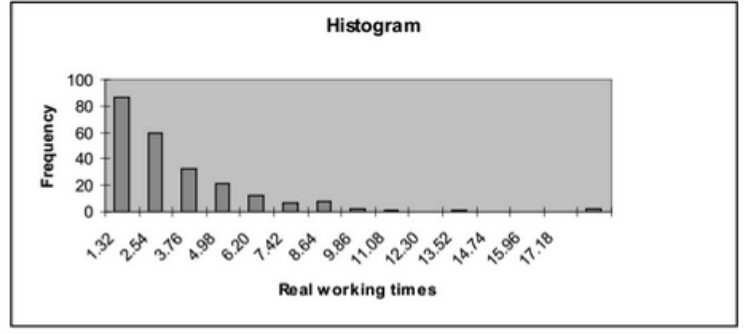

b)

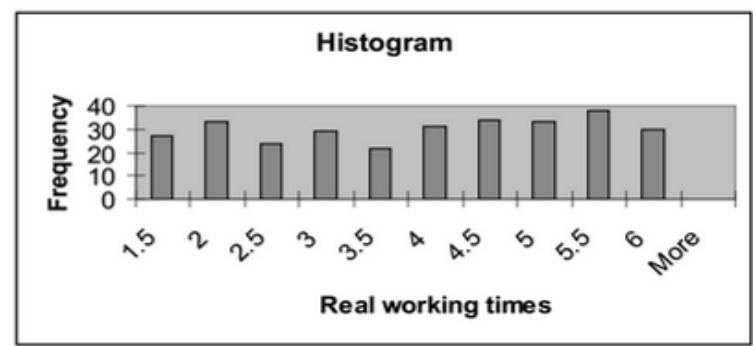

c)

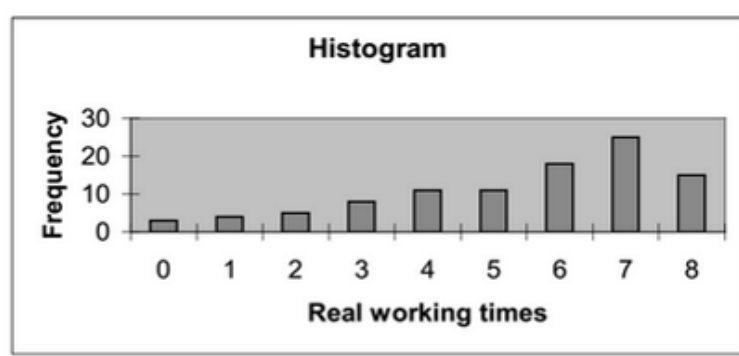

d)

Fig. 1. Types of statistical distributions used
The next step is to introduce the model created in the SimQuick simulator in Excel. For each element in the model, a table will open, pressing the element button, containing all the details.

Below is an example of the control panel generated by SimQuick related to the simple model of the queue at the bank counter, for an easier understanding of the simulation mechanism with this program (Fig. 2).

There are 10 different ways to customize the time duration between 2 inputs (generating a normal distribution around a given value and a set margin of an exponential distribution, or a constant uniform distribution, defining various time scenarios or entering given intervals).

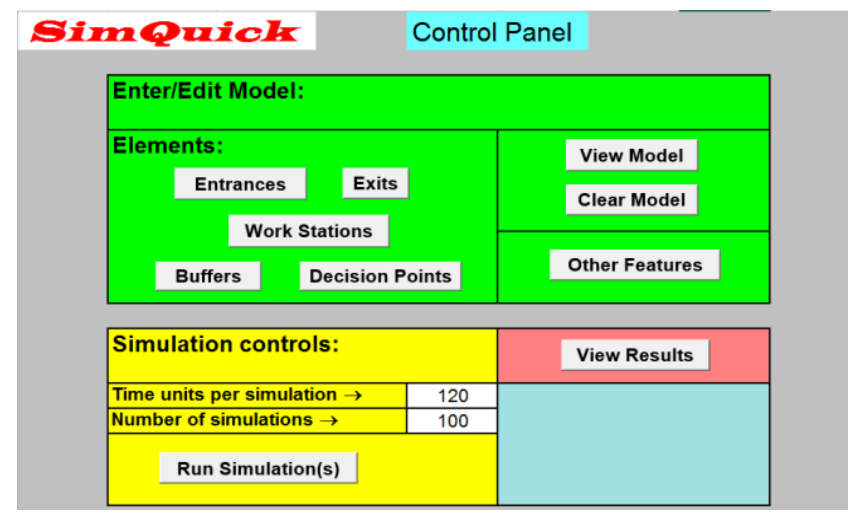

a)

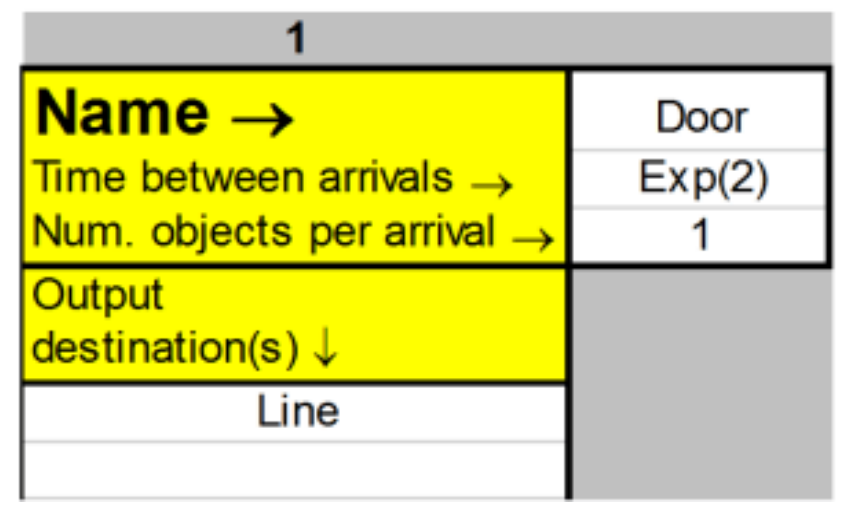

b)

Fig. 2. Data entry interface model in the inputs section for the process logic diagram

The aim is to give the possibility to choose a scenario as close as possible to the simulated process.

\section{Process simulation - before and after the implementation of changes}

The simulation involves the introduction of the mathematical model, previously defined, in the simulation software and its running, once determined all the necessary characteristics (number of relevant simulations, duration of a simulation, etc.), both for the process that works BEFORE and AFTER the implementation of changes.

The results obtained from the simulations are presented in the form of Excel tables. It is up to the 
users of the simulation the graphical or analytical way in which these results are presented. In figure 3 there are two examples - one tabular and one graphical, of how to evaluate the results. We considered as parameter to be analyzed the response lead time to a customer request. The simulation is showing before/after the result improved from 5 weeks to 1 week. The simulation is not giving the solution but it is providing the quantifiable data based on what decisions can be made. In this case the producer can move from batch production to JIT production with minimal inventory and fast responses to customer requests.

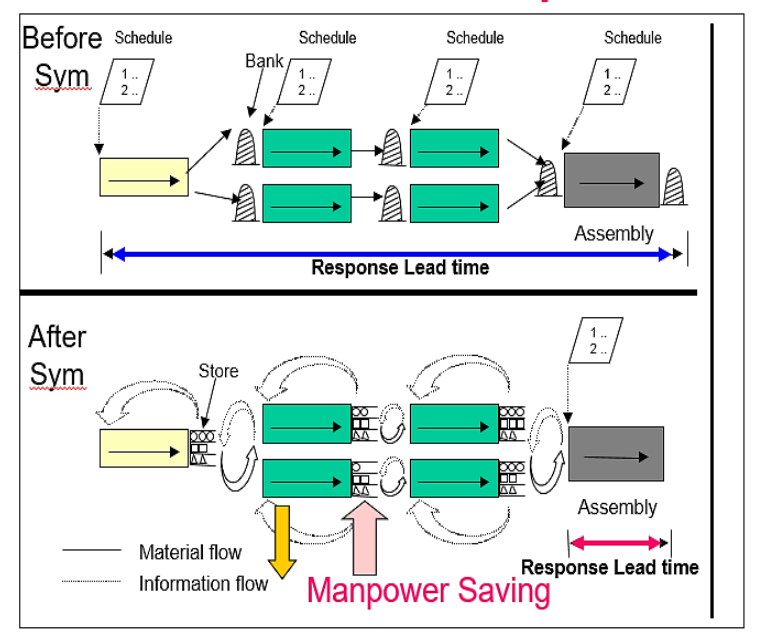

Fig. 3. Modeling the process BEFORE and AFTER implementing the changes

In the simulation results in figure 4 we can observe another simulation output: manpower utilization. Based on the initial results the user can take the necessary measures:

- Rebalance the line;

- Eliminate rework or over processing activities;

- $\quad$ Adjust the speed of inline equipment;

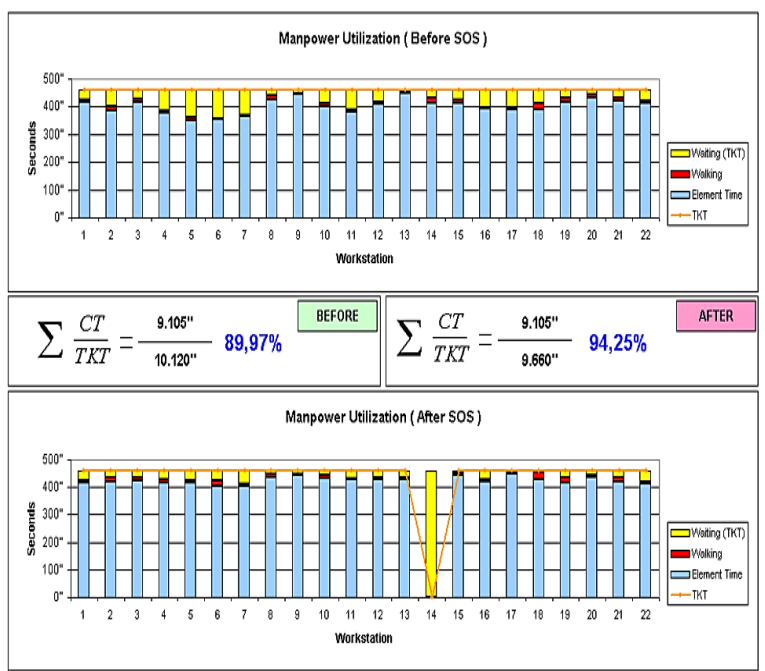

Fig. 4. Before and after results for manpower utilization
In order to reduce the downtime in the "before" simulation case we observe:

- $\quad$ Long lead time (large order lot);

- Difficult to change schedule;

- Cannot see the pace;

- Cannot see when to stop work extra hours, extra equipment;

In the "after" simulation case we observe:

- Flexible for schedule change;

- Short lead time;

- Clear informational

- Can see when to stop

- Can see idle areas;

- Take out extra hours / equipment;

A line production output per article/week, simulation done in a harness production corporation (Fig.5) is leading to another improvement plan:

- Level scheduling;

- Implementation of Kanban delivery system;

- Inventory control management in TTM;

- Reduce scrap;

- Reduce distance of travel;

- Standardize bill of materials.
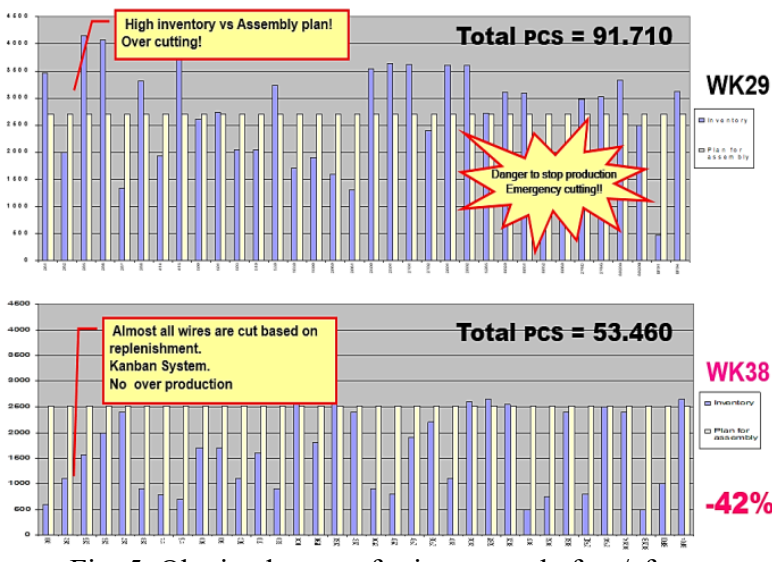

Fig. 5. Obtained output for inventory before/after

We observe the results after 9 weeks. Even if the output is reduced to $60 \%$ the demand is met and the equipment for cutting are cutting only what is needed.

In order to have an accurate simulation, we need to have reliable and accurate data as input, and a clear algorithm as method. This role is delivered by the traceability (TTM). 


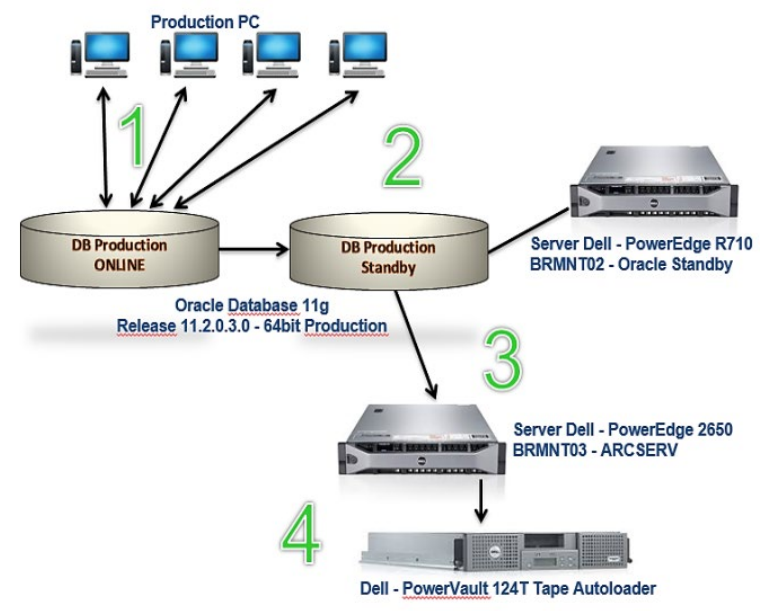

Fig.6. TTM Architecture

The data gathering is done through production PCs and the algorithm is developed based on the needs, an example from a steering wheel producer in figure 5 .

The description of the TTM architecture is presented in figure 6 :

1 - Active connection between clients and Oracle DB. Primary connection opened from clients;

2 - Standby DB (database). A standby database is a database replica created from a backup of a primary database. By applying archived redo logs from the primary database to the standby database, one can keep the two databases synchronized;

3 - Backup ARCSERVCA ARCserve ${ }^{\circledR}$ Backup for Windows;

4 - Backup on tape.

For instant data acquisition is mandatory that the equipment to be connected to the TTM (Fig.7). Also with additional features TTM is able to adapt and to provide instant simulation based on requested algorithms. In our case the data for the simulation is gathered from the equipment's 5 workstations. Each workstation is having the TTM software installed and for each product the technical parameters are saved per his unique ID.

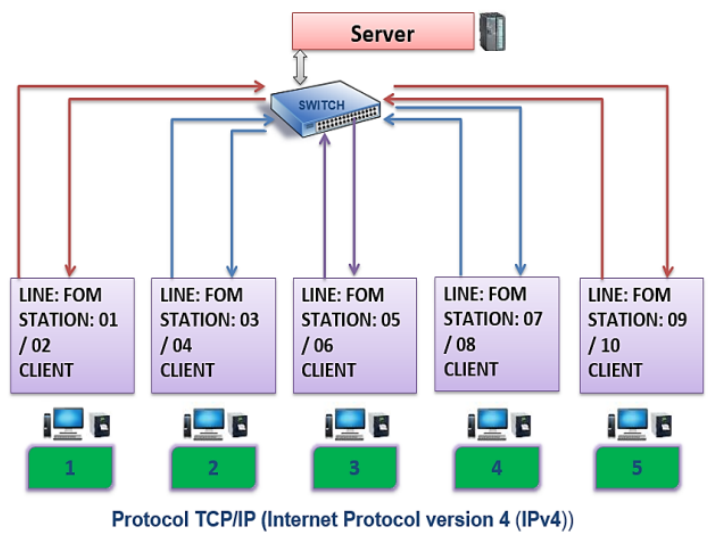

Fig.7. Equipment Connection to Traceability
All the data is continually backed up on the server and transformed for the human interface needs (production reports, technical reports, logistic and quality reports).

When the analysis of the simulation data is done the root causes of variation are divided in 2 categories:

- Special Causes;

- Common Causes.

The correction, elimination or minimization of these two types of variation require different actions:

- Statistical rules are used to identify special causes, or root cause analysis methodologies (Ishikawa, 8D, and Shainin Problem Solving);

- When only common cause variation exists, a process is "in control".

Special Cause Variation is assignable to events. In order to eliminate it, the user must isolate and address those events.

Action should be taken to prevent the cause form occurring again; to at least to mitigate the effect of the event that should it happen in the future.

Common Cause Variation is the absence of special causes. It is naturally occurring, random variation and every process has some magnitude of variation. It is important not to react to common cause as it is expected given the natural variation in the process or product.

For a correct simulation the user must eliminate first the special causes. If failed to do so the result of the simulation is irrelevant. The common cause will provide variation in control, but may not fulfill customer expectations.

Common cause variation can only be affected through fundamental product or process changes.

\section{Conclusion}

The results obtained from the simulations are presented in the form of Excel tables. It is up to the users of the simulation the graphical or analytical way in which these results are presented.

The authors of this article have conceived, designed, implemented traceability software at an enterprise in the automotive field, and the specific components. This software is able to perform various orders at the enterprise level including simulations of the production process.

The simulation is done using the SimQuick program when necessary and is controlled semiautomatically. SimQuick is a complementary tool to TTM, with the purpose of providing data which be consequently analyzed by a user. The entire operation is coordinated by the traceability program, which conducts a lot of vital functions under the generic name of TTM (Total Traceability Management).

A powerful simulation tool can be developed based on artificial intelligence (AI), as a module to TTM, in instant link, providing "big data" analysis capability in order to forecast and preventively simulate the variation of the key performance output indicators, 
alerting the user that an error has an increased probability of occurrence. Forecasting tendencies will be the key success factor for future automated business in terms of predictive maintenance, market evolution and human behavior in manufacturing conditions.

\section{References}

[1] Bărbosu, D. and Zelina, I. (1998), Probabilities Calculation, Editure CUB PRESS22, Baia Mare, Roamnia.

[2] Harvigssen, D. (2016), Process simulation with Excel, 3rd edition, University of Notre Dame.

[3] Simul 8 - Simulation Software. [Online]. Available: https://www.simul8.com.

[4] Ambrus, O. (2018), Contribution to the application of lean manufacturing systems in the automotive industry, $\mathrm{PhD}$ Dissertation, Oradea University, Romania.

[5] Voehl, Fr. (2013), The Lean Six Sigma Black Belt Handbook: Tools and Methods for Process Acceleration, CRC Press.

[6] Defeo, J.A (2016), Juran's Quality Handbook: The Complete Guide to Performance Excellence, Seventh Edition, McGraw-Hill Education; 7th edition.

[7] Brunner, F.J. (2017), Japan's Success Concept, KAIZEN, KVP, Lean Production Management, Total Productive Maintenance Shopfloor Management, Toyota Production System, GD ${ }^{3}$ Lean Development (Praxisreihe Qualität), Carl Hanser Verlag GmbH \& Co. KG.

[8] Mcloughlin, C. and Miura T. (2017), True Kaizen: Management's Role in Improving Work Climate and Culture, Productivity Press, USA.

[9] Wilson, L. (2017), How to implement Lean Manufacturing, 2nd edition McGraw Hill, USA.
[10] Mrugalska, B. and Ahmed, J. (2021), Organizational agility in industry 4.0: A systematic literature review, Sustainability, 13(15), 8272.

[11] Jing, L., Baoping, C. and Wang, L. (2021), Prognostic and health management through collaborative maintenance, Journal of manufacturing systems, Epub ahead of print.

[12] Murphy, N.R. (2016), Site Reliability Engineering: How Google Runs Production Systems, 1st edition, O'Reilly Media.

[13] Su, C.T., Chiang, T.L. and Chang, C.M. (2006) Improving service quality by capitalizing on an integrated Lean Six Sigma methodology, International Journal of Six Sigma and Competitive Advantage, vol. 2(2), pp. 1-22.

[14] Debashis, S. (2007), Lean for Service Organizations and Offices. A Holistic Approach for Operational Excellence, ASQ Quality Press.

[15] Shewhart, W.A. (1986), Statistical Method from the Viewpoint of Quality Control, $1^{\text {st }}$ edition, Dover Publications

[16] Pyzdek, T. and Keller, P.(2013) - The handbook for Quality management- a complete guide for operational excellence, 2nd edition, McGrawHill Education.

[17] Taiichi, O. (1988), Toyota Production System: Beyond Large-Scale Production, Productivity Press, Portland, Oregon, USA.

[18] Rother, M. and Shook, J. (2003), Learning to See - value-stream mapping to create value and Eliminate Muda, 1st edition, Amazon.

[19] Tennant, G. (2017), Six Sigma: SPC and TQM in Manufacturing and Services, 1st edition, Routledge, London. 